\title{
石墨烯基分离膜可行吗?
}

孙成珍*，白博峰

西安交通大学动力工程多相流国家重点实验室, 西安 710049

* 联系人, E-mail: sun-cz@xjtu.edu.cn

\section{Are graphene-based membranes practicable?}

\author{
Chengzhen Sun ${ }^{*} \&$ Bofeng Bai
}

State Key Laboratory of Multiphase Flow in Power Engineering, Xi'an Jiaotong University, Xi'an 710049, China

* Corresponding author, E-mail: sun-cz@xjtu.edu.cn

doi: 10.1360/TB-2019-0515

提高膜材料的分子渗透速率对于膜分离技术在气体 分离和水净化等领域的高效应用至关重要. 通常认为, 膜 的渗透率与其厚度成反比例关系. 因此, 石墨烯及其衍生 物由于原子尺度的厚度成为了理想的分离膜材料. 从石墨 烯被发现开始，石墨烯基分离膜是否可行的问题就成为了 人们关注和研究的热点.

目前, 两种结构和机理完全不同的石墨烯基分离膜 概念被提出: 一是具有纳米级孔隙的多孔石墨烯, 二是具 有层状结构的氧化石墨烯. 完整的石墨烯材料对分子和离 子都具有不可渗透性 ${ }^{[1]}$, 但当产生纳米级的孔隙后, 多孔 石墨烯可以基于分子和离子的尺寸篮选效应实现气体分 离和水净化(图1(a)). 氧化石墨烯是具有很多含氧功能团 的石墨烯层状材料, 利用层间纳米通道和石墨烯上的固有 缺陷等可以实现分子和离子的选择性渗透(图1(b)). 理论 上, 多孔石墨烯和氧化石墨烯都可以作为气体分离和水净 化的分离膜, 但还需要很多努力才能让它们在实际工业过 程中得到应用. 由于渗透机理和膜厚度不同, 多孔石墨烯 的渗透率远远大于氧化石墨烯. 然而, 多孔石墨烯分离膜 的制备过程比氧化石墨烯分离膜复杂. 多孔石墨烯膜的制 备涉及石墨烯的转移、纳米孔的产生等繁琐过程, 并且还 存在很多问题; 而氧化石墨烯膜可以简单地通过真空抽 滤、旋涂等方法实现. 也就是说, 多孔石墨烯分离膜的优 点是高渗透率, 而氧化石墨烯分离膜的优点是易于大面积 制备. 因此, 人们一直追求一种既具有高渗透率又易于大 面积制备的新型石墨烯基分离膜结构.

目前, 已有很多实验测量研究证实了石墨烯基分离 膜确实可以实现气体分离和水净化. 2012年, Koenig等人 ${ }^{[2]}$ 通过紫外辐射氧化制备了微米级别面积的多孔石墨烯材 料, 并成功测试了其不同气体分子的选择性渗透特性. 随
后，厘米级别面积的多孔石墨烯膜被成功制备，并被证明 可用于气体分离和水净化 ${ }^{[3 \sim 5]}$, 大大促进了多孔石墨烯分 离膜的发展. 学者们甚至已经找到了能够精确控制纳米孔 尺寸的办法 ${ }^{[6]}$. 大面积的氧化石墨烯也同样被证实可实现 高效的气体分离和水净化 ${ }^{[7 \sim 9]}$. 尽管石墨烯基分离膜已经 被证实在实验室可行，但是在实际应用过程中还会遇到很 多挑战, 包括高机械强度和无缺陷大面积石墨烯基分离膜 的制备、尺寸和化学组分精确控制的高密度纳米孔的产生 以及一些常见的其他问题，比如支撑、阻塞、积垢和浓差 极化等.

最近, 武汉大学袁荃教授课题组和加州大学洛杉矶 分校段镶锋教授课题组 ${ }^{[10]}$ 合作制备了基于多孔石墨烯和 单壁碳纳米管的复合薄膜, 并研究了其海水淡化性能(图 2(a))．这种复合膜的制备涉及单壁碳纳米管网络向石墨烯 表面的转移、介孔二氧化硅薄膜在石墨烯表面的生长、基 于氧等离子体的纳米孔产生以及二氧化硅薄膜的移除等 过程(图2(b)). 通过电子显微镜观测, 可以看出很明显的碳

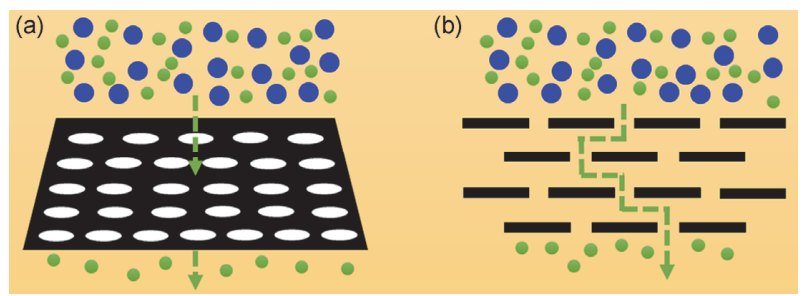

图 1 (网络版彩色)石墨烯基分离膜的原理. (a) 多孔石墨烯分离膜 原理示意图; (b) 氧化石墨烯分离膜原理示意图

Figure 1 (Color online) Schematic illustration of graphene-based membranes. (a) Principle of nanoporous graphene membranes; (b) principle of graphene oxide membranes 
(a)
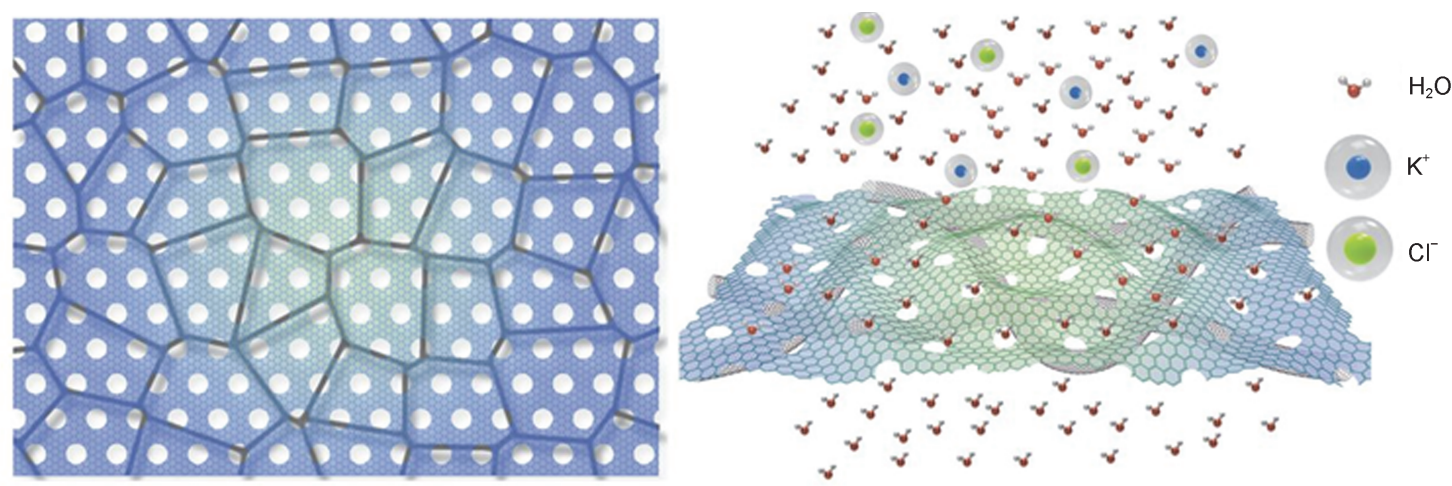

(b)
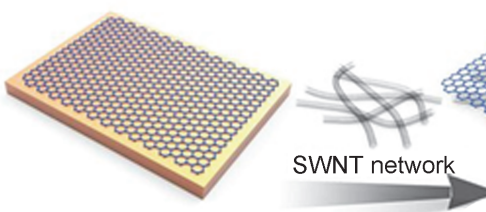

Graphene on $\mathrm{Cu}$

(c)

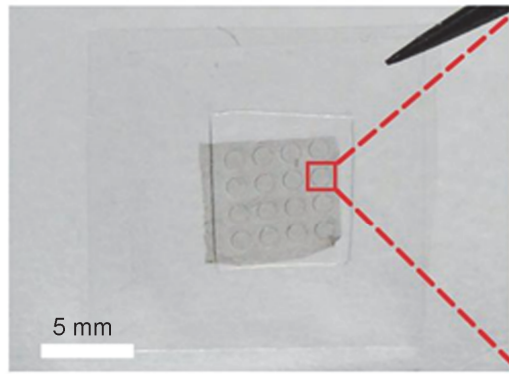

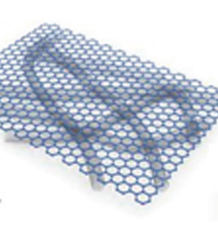

Graphene/SWNT

membrane

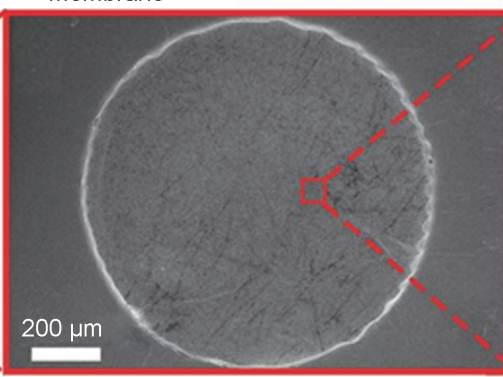

$\mathrm{O}_{2}$ plasma

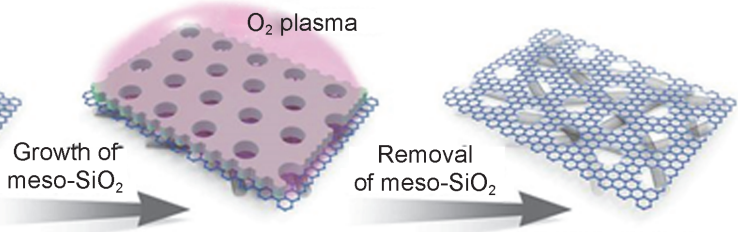

GNM/SWNT

membrane

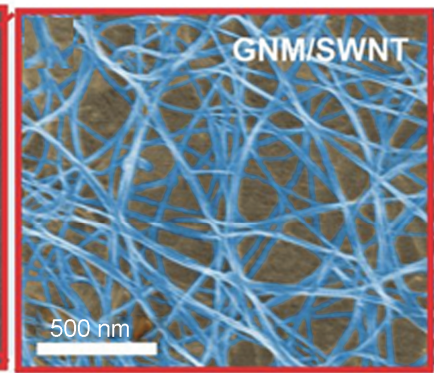

图 2 (网络版彩色)石墨烯基复合分离膜. (a) 基于复合膜的海水淡化原理示意图; (b) 复合膜的制备过程示意图; (c) 复合膜的实物图和 扫描电子显微镜图 ${ }^{[10]}$

Figure 2 (Color online) Graphene-based hybrid membranes. (a) Schematic illustration of the hybrid membranes for water desalination; (b) fabrication process of the hybrid membranes; (c) photo and scanning electron microscopy images of the hybrid membranes ${ }^{[10]}$

纳米管网状结构(图2(c)). 他们发现, 这种薄膜具有很高的 水渗透通量和离子排斥率, 这是由石墨烯上引人的高密度 选择性纳米孔导致的. 同时, 由于碳纳米管的支撑作用, 这种复合膜具有很好的结构完整性和机械强度.

通过分析很容易看出, 这种复合膜同时具备了多孔 石墨烯膜和氧化石 墨烯膜的优点: (1) 具有由石墨烯纳米 孔带来的高渗透率; (2) 碳纳米管网络的连接作用使其容 易大面积制备. 值得注意的是, 这种新型的复合膜也克服
了石墨烯基分离膜当前面临的最大挑战—制备同时具有 高密度纳米孔和高机械强度的大面积石墨烯基薄膜. 该工 作发表在S cience 上 $^{[10]}$. 该研究进一步说明, 通过逐步克服 当前面临的困难和挑战，石墨烯基分离膜确实是可行的， 并且能够最终应用到实际工业过程中. 我们希望通过科学 家和工程师们持续不断的努力, 不仅可以使二维的石墨烯 基分离膜从概念变为现实, 而且能够促进石墨烯薄膜在能 源转换与利用、生物工程等领域的广泛应用.

\section{参考文献}

1 Bunch J S, Verbridge S S, Alden J S, et al. Impermeable atomic membranes from graphene sheets. Nano Lett, 2008, 8: 2458-2462

2 Koenig S P, Wang L D, Pellegrino J, et al. Selective molecular sieving through porous graphene. Nat Nanotechnol, 2012, 7: 728-732

3 Celebi K, Buchheim J, Wyss R M, et al. Ultimate permeation across atomically thin porous graphene. Science, 2014, 344: 289-292

4 O'Hern S C, Boutilier M S H, Idrobo J C, et al. Selective ionic transport through tunable subnanometer pores in single-layer graphene membranes. Nano Lett, 2014, 14: 1234-1241 
5 Surwade S P, Smirnov S N, Vlassiouk I V, et al. Water desalination using nanoporous single-layer graphene. Nat Nanotechnol, 2015, 10: 459-464

6 Zhao J, He G, Huang S, et al. Etching gas-sieving nanopores in single-layer graphene with an angstrom precision for high-performance gas mixture separation. Sci Adv, 2019, 5: eaav1851

7 Joshi R K, Carbone P, Wang F C, et al. Precise and ultrafast molecular sieving through graphene oxide membranes. Science, 2014, 343: $752-754$

8 Li H, Song Z, Zhang X, et al. Ultrathin, molecular-sieving graphene oxide membranes for selective hydrogen separation. Science, 2013, 342: 95-98

9 Nair R R, Wu H A, Jayaram P N, et al. Unimpeded permeation of water through helium-leak-tight graphene-based membranes. Science, 2012, 335: 442-444

10 Yang Y, Yang X, Liang L, et al. Large-area graphene-nanomesh/carbon-nanotube hybrid membranes for ionic and molecular nanofiltration. Science, 2019, 364: 1057-1062 